\title{
A SIMPLE FAIR CELL DISCARDING ALGORITHM ON PER-VC QUEUEING BASIS
}

\author{
Xiaowen Mang and Yonatan Levy \\ ATET Labs \\ 200 Laurel Ave, Middletown, NJ 07748, USA \\ $\{x m a n g, y l e v y\}$ Qatt.com
}

\begin{abstract}
In this paper we propose a simple Fair Cell Discarding (FCD) algorithm with virtual per-VC queueing. The main objective of FCD algorithm is to redistribute the cell losses according to each connection's QoS requirement. In case the buffer is full, the newly arrived cell is not automatically discarded but rather a cell from a sub-queue $U$ which is selected according to a fairness criterion which is defined by connections mean arrival rates and their QoS (Quality of Service) such as CLR (Cell Loss Ratio) requirements. Extensive analytical analysis and simulations have been conducted to evaluate FCD algorithm's property and to validate its effectiveness under various traffic and switch configurations. Our study reveals that a very simple approach like FCD can actually differentiate connections with different traffic characteristics and QoS requirements; and satisfy their specified QoS as long as a minimum amount of resources (bandwidth and total buffer space) is allocated for guaranteeing the aggregate QoS/CLR requirement of all active connections.
\end{abstract}

Keywords: ATM Neworks, quality of service, per-VC queueing, fairness, selective cell discard.

\section{INTRODUCTION}

ATM networks have become a reality. However, how to successfully provide a specified QoS (Quality of Service), and in particular how to differentiate connections with different QoS requirements, is an important open issue which will impact the success of ATM technology in 
heterogeneous network environments and yet is far from a satisfying resolution.

In this paper, we argue that in order to guarantee individual connection's QoS, a Per-VC queueing architecture should be used, and intelligence must be added to the cell discarding process. We tackle the issue by proposing a Fair Cell Discarding (FCD) algorithm with virtual per-VC queueing.

In the past, much effort has been spent focusing on methods for increasing network efficiency by maximizing statistical gain while still guaranteeing connections QoS, and most of that work is based on a single FIFO (First In First Out) buffer for all connections. The obvious problem with this approach is that there is no way that one can distinguish one connection from another without adding some costly enhancement. A previous study (Mang et al., 1996) shows that in a single FIFO queueing system, if the cells are dropped upon arrival in case of buffer overflow, for a given set of connections, the burstier connections experience a higher CLR (Cell Loss Ratio) and actually exceed their QoS threshold, while the QoS of aggregate traffic is still satisfied.

In contrast to a single FIFO queueing architecture, per-VC queueing provides a more flexible and natural architecture for enhancing traffic management at ATM switches. Assuming per-VC queueing architecture at the ATM switch, which is already a reality, one needs to decide how to fairly distribute shared resources across active connections in order to provide QoS assurance to individual (or groups of) connections. Resource re-distribution can be in various forms, i.e. service scheduling (Parekh et al., 1993) (Golestani, 1994)(Lee et al., 1994)(Demers /em et al. 1989) (Archambault et al., 1996), buffer management (Choudhury et al., 1996)(Collier et al., 1996) (Takagi et al., 1991) and selective cell discarding (Yang et al., 1996) (Conway et al., 1996)(Kawahara et al., 1996) (Chen et al., 1996)(Heyman et al., 1992)(Wilson et al., 1996) at moments of resource scarcity. Service scheduling primarily deals with how to fairly allocate available bandwidth to a set of connections. It regulates queue length distribution for each logical sub-queue, but has no control over which cell should be dropped or discarded in the event of buffer overflow. This in turn is handled through buffer management and selective cell discarding schemes. They both control access to the buffer space.

A variety of buffer sharing strategies is summarized and analyzed in (Collier et al., 1996) under non-uniform bursty traffic. Some well known strategies are complete partitioning, complete sharing, partial sharing, sharing with minimum allocation, sharing with maximum queue length, and fair sharing. All these sharing schemes require some pre-calculated 
threshold values, which demand prior knowledge of traffic characteristics and of the service discipline to be used, and, therefore, are very difficult to determine. In (Choudhury et al., 1996), a dynamic queue length thresholds scheme is proposed to selectively drop packets where the maximum permissible length for any individual queue at any instant of time is a function of the unused buffering in the switch. Compared with the static threshold scheme, this scheme automatically adapts to load variation among output queues, and hence it is more robust. However, the assurance of cell loss requirements for individual connections is not addressed in any of the above schemes.

A weighted fair blocking (WFB) mechanism for discrete-time multiplexing is proposed in (Conway et al., 1996). WFB rejects some of the packets in a batch arrival when there is an insufficient number of available buffers. Although it is claimed that WFB decouples buffer dimensioning from parameterization of the discard mechanism, the process of determining appropriate weights so as to satisfy different loss requirements in a heterogeneous traffic environment remains as difficult as determining appropriate threshold values in any threshold based control policies. The fact that linear programming approach is used in the calculation of the packet selection probabilities and prior knowledge of source traffic characteristics is required further prevents WFB from being a practical solution.

In (Heyman et al., 1992)(Yang et al., 1996) the cell loss performanc e of individual connections is addressed through selective discarding. In (Heyman et al., 1992), the ATM multiplexer keeps track of accumulated cell losses for all active connections. When a new cell arrives, if the buffer is full that cell is not automatically dropped. Instead, a cell from a connection that has the lowest current accumulated loss rate (among all connections that have cells currently in the buffer) is dropped. The arriving cell is dropped only if its connection has the lowest loss rate so far. This approach is based on the homogeneous traffic assumption. Since it requires on-line measurements of both number of losses and arrivals, this approach can only remain as an illustration of how selective discarding affects cell loss performance of individual connections rather than

a practical solution. A generalized version of the above, a "QoSscheme", is given in (Yang et al., 1996) to deal with situations where traffic stre ams have different traffic characteristics as well as different loss (QoS) requirements. When a cell arrives and the buffer is full, a cell is discarded only if it belongs to a connection with the smallest ratio between its loss ratio measurement and its loss requirement, a predefined number. The limitations of this QoS-scheme are that on-line 
measurements of cell loss ratio are required, and it is not effective if all connections are not equally demanding.

The main objective of the FCD algorithm we propose here is to redistribute cell losses according to each connection's QoS requirement. The model we consider in our study is an ATM statistical multiplexer with a complete shared output port buffering. FCD employs a fairness criterion defined as a function of traffic load and CLR requirements. We define $L_{x}^{\text {fair }}$ as connection $x$ 's Fair Ratio of Loss (FRL):

$$
L_{x}^{f a i r}=\frac{\lambda_{x} p_{x}}{\sum_{x=1}^{N} \lambda_{x} p_{x}}
$$

here $\lambda_{x}$ stands for the mean arrival rate of connection $x, N$ is the total number of active connections, and $p_{x}$ is connection $x$ 's CLR requirement . FCD employs an approach similar to one suggested in (Heyman et al., 1992)(Yang et al., 1996) where, in case the buffer is full, the newly arriving cell is not automatically discarded but rather a cell from a subqueue $u$ which is selected according to the fairness criterion $L_{u}^{\text {fair }}$. Here, for the first time, we provide an explicit implementation for how to select and discard cells. In the above existing methods only observations and selective discard principles are reported. None of those proposals provide the mechanism to deal with the situation where the newly arriving cell does not belong to the selected connection or the subqueue of the selected connection is empty. By introducing a Bargain Vector, we provide a very effective and practical way to handle this situation. If the selected sub-queue $u$ is empty, a cell can be discarded from another non-empty sub-queue. This event is recorded by a Bargain Vector $\mathbf{B}$. The reason we call $\mathbf{B}$ a Bargain Vector is that it keeps a record of loss exchange between connections. At time $t_{1}$ connection $u$ may lose a cell for connection $v$, and at $t_{2}$ connection $w$ may lose a cell for connection $u$, and so forth. The content of Bargain Vector B is a set of integers. For $n>0, B_{u}=n$ means that $n$ cells were discarded from connection $u$ because other selected subqueues were empty, while $B_{u}=-n$ means that $n$ cells were discarded from other sub-queues because the selected sub-queue $u$ was empty. In the long run, the use of Bargain Vector $\mathbf{B}$ further enhances the fairness preserved by FRL of 1.1 .

In our proposed scheme, per-VC queueing architecture can be generalized into per-class queueing. A class is a group of VCs which have similar traffic characteristics and QoS requirements. Such generalization tackles the concerns when there are thousands of VCs. Current (available on the market) ATM switches support up to $12 \mathrm{~K} \mathrm{VC/VPs.}$

We describe the FCD algorithm and discuss its properties in Section 2. We devote Section 3 to validating the effectiveness of FCD under various 
traffic scenarios and switch configurations. In Section 4, we conclude our study and highlight our findings. Mathematical proofs are given in the Appendix.

\section{FAIR CELL DISCARDING ALGORITHM $2.1 \quad$ FCD DESCRIPTION}

The model we consider in our study is a complete buffer sharing scheme, where cells from different connections form logical sub-queues. Let $Q_{x}$ be the sub-queue length of connection $x$ and $S$ be the buffer size, the FCD algorithm is given as follow:

Intialize:

$N$ is the number of active connections;

For $x=1: N$ Calculate $L_{x}^{f a i r}, B_{x}=0$

After a call is accepted:

$N=N+1$;

For $x=1: N$ Calculate $L_{x}^{\text {fair }}$

When a cell of connection $w$ arrives

1. IF $\sum_{x} Q_{x}=S$ (Buffer is full)

(a) Select $u \in\{1, N\}$ according to $L_{u}^{\text {fair }}$

- Option I - Deterministic selection

- Option II - Probabilistic selection

(b) IF $Q_{u}=0$ or $B_{u}>0$

Choose $v$ by $B_{v}=\min \left\{B_{x} ; Q_{x}>0\right\}$

Update B: $B_{v}=B_{v}+1$ and $B_{u}=B_{u}-1$

ELSE

$v=u$

(c) IF $v=w$

Discard the newly arrived cell

ELSE

Discard a cell from sub-queue $v$

Put newly arrived cell in the sub-queue $w$

2. ELSE (Buffer is not full)

Put newly arrived cell in the sub-queue $w$ 
In Table 1.1 we demonstrate how the values of $\mathbf{B}$ can be updated for the case of 3 connections. The total buffer size is $S=60($ cells $)$ and $t_{n}$ stands for the instant of the $n$th example. If a connection is selected at $t_{n}$, its corresponding queue length is underlined. If a connection's queue length or its bargain vector value is updated at $t_{n}^{+}$, the updated value is highlighted in bold. Note that after the update, the total number of cells in the buffer is 59 , leaving room for the new cell.

Table 1.1 How FCD Works

\begin{tabular}{llllll}
\hline & $t_{1} / t_{1}^{+}$ & $t_{2} / t_{2}^{+}$ & $t_{3} / t_{3}^{+}$ & $t_{4} / t_{4}^{+}$ & $t_{5} / t_{5}^{+}$ \\
\hline$Q_{1}$ & $\underline{28} / \mathbf{2 7}$ & $55 / 55$ & $\underline{0} / 0$ & $15 / 15$ & $\underline{6} / \mathbf{5}$ \\
$B_{1}$ & $0 / 0$ & $0 / 0$ & $-3 /-4$ & $1 / 1$ & $0 / 0$ \\
& & & & & \\
$Q_{2}$ & $2 / 2$ & $\underline{0} / 0$ & $60 / \mathbf{5 9}$ & $20 / \mathbf{1 9}$ & $40 / 40$ \\
$B_{2}$ & $0 / 0$ & $0 /-1$ & $5 / \mathbf{6}$ & $-2 /-1$ & $-1 /-1$ \\
& & & & & \\
$Q_{3}$ & $30 / 30$ & $5 / 4$ & $0 / 0$ & $\underline{25} / 25$ & $14 / 14$ \\
$B_{3}$ & $0 / 0$ & $0 / 1$ & $-2 /-2$ & $1 / 0$ & $0 / 0$ \\
\hline
\end{tabular}

The above table can be further explained as following:

- $t_{1}$ : Selected $Q_{1}>0, B_{1}=0$; discard one cell from $Q_{1}$.

- $t_{2}$ : Selected $Q_{2}=0$; update $B_{2}$ and $B_{3}$; discard one cell from $Q_{3}$.

- $t_{3}$ : Selected $Q_{1}=0 ;$ update $B_{1}$ and $B_{2}$; discard one cell from $Q_{2}$.

- $t_{4}$ : Selected $Q_{3}>0, B_{3}>0, B_{2}=\min \left(B_{1}, B_{2}, B_{3}\right)$; update $B_{2}$ and $B_{3}$; discard one cell from $Q_{2}$.

- $t_{5}$ : Selected $Q_{1}>0, B_{1}=0$; discard one cell from $Q_{1}$

There are two alternatives of implementing selection process of step 1(a) in the above FCD algorithm:

Option I - Deterministic implementation. Connection $u$ is obtained in a Round Robin fashion from a Discard Table (DT) whose entries are integers of $\{1, N\}$ representing active connections. A simple generalized round robin algorithm for constructing DT based on the vector $\left\{L_{x}^{\text {fair }}\right\}$ is given in (Arian at al., 1992). The algorithm is optimal for $N=2$ and pseudo-optimal for $N>2$. The DT has to be recomputed whenever a connection is added or terminated. 
Option II - Probabilistic selection. Connection $u$ is obtained according to the distribution $\left\{L_{u}^{\text {fair }}\right\}$ using a random number generator.

\subsection{PROPERTIES OF FCD}

We now state some theoretical properties of the FCD algorithm. Proofs are given in the Appendix.

Property I. For given total buffer size and total bandwidth, the FCD algorithm is path-wise equivalent (in terms of total cell losses) to complete buffer sharing schemes where cell dropping occur upon cell arrival.

Property II. For given buffer size and bandwidths $C_{1}>C_{2}$, the resulting CLRs (Cell Loss Ratios) satisfy

$$
\frac{p_{1}\left(C_{1}\right)}{p_{1}\left(C_{2}\right)}=\frac{p_{2}\left(C_{1}\right)}{p_{2}\left(C_{2}\right)}=\ldots=\frac{p_{N}\left(C_{1}\right)}{p_{N}\left(C_{2}\right)}<1
$$

Properties I and II do not claim that FCD controls the aggregate loss performance, but rather that it re-distributes the losses across connections in a fair manner. A direct benefit from Propertys I and II is that FCD adds more freedom to the CAC (Connection Admission Control) process and reduces buffer management overhead. In FCD one can have both,the efficiency of complete shared buffering and the performance advantages of dedicated buffering. The latter is achieved through selective discarding where a new arrival is not automatically rejected when the buffer is full.

Property III. If a subset of connections $\mathbf{V} \subseteq \mathbf{U}$ submits traffic at a lower mean rate, $\lambda_{v}^{*}$, than their declared rate, $\lambda_{v}$, i.e. $\lambda_{v}^{*}<\lambda_{v}$, then the resulting CLRs satisfy

(a) $\quad p_{u}^{*}<p_{u}, \quad \forall u \in\{1, N\}$.

and

$$
\text { (b) } \quad \frac{p_{w}^{*}}{p_{w}}<\frac{p_{v}^{*}}{p_{v}}, \quad \forall w \notin \mathbf{V} \text { and } \forall v \in \mathbf{V}
$$

Property III implies that if a connection $u$ is submitting traffic at a lower rate than what it declares, all active connections' loss rates are lower; however, this particular connection $u$ 's normalized CLR is higher than the others. We define "normalized CLR" as $\frac{\text { actual } C L R}{C L R \text { requirement }}$. Also from Property II we note that if all active connections are submitting traffic at their declared rates, FCD guarantees their normalized CLR to be equal. 
Property IV. If a subset of connections $\mathbf{V} \subseteq \mathbf{U}$ submits traffic at a higher mean rate, $\lambda_{v}^{*}$, than their declared rate, $\lambda_{v}$, i.e. $\lambda_{v}^{*}>\lambda_{v}$, then the resulting CLRs satisfy

$$
\text { (a) } \quad p_{u}^{*}>p_{u}, \quad \forall u \in\{1, N\} .
$$

and

$$
\text { (b) } \frac{p_{w}^{*}}{p_{w}}>\frac{p_{v}^{*}}{p_{v}}, \quad \forall w \notin \mathrm{V} \text { and } \forall v \in \mathbf{V}
$$

Property IV states that if some connections violate their traffic contracts, all connections' QoS will be degraded. In order to protect conforming sources from violating sources, a misbehaved traffic will be tagged by UPC (Usage Parameter Control) and in case of buffer overflow, arriving tagged cells are discarded first before FCD is applied.

\section{$2.3 \quad$ ROBUSTNESS OF FCD}

Regarding the use of the bargain vector, a question one may ask is whether $\mathbf{B}$ needs to be reset periodically. If connection $u$ is silent for $\Delta T$ period, then $\left|B_{u}(\Delta T+t)-B_{u}(t)\right| \leq p_{u} \lambda_{u} \Delta T$, and the time needed to accumulate one debt will be

$$
T_{s} \geq \frac{1}{p_{u} \lambda_{u}}
$$

Assume that most of applications' mean rates are in the range of megabits per second $(1 \mathrm{Mbps}=2359 \mathrm{cells} / \mathrm{sec})$, and CLR requirements are around $10^{-7}$, it is safe to say that $T_{s}$ is around 4240 seconds. For instance if a connection is idle for more than one hour and its mean rate is $1 \mathrm{Mbps}$ and its CLR requirement is $10^{-7}$, we have $\mid B_{u}(1$ hour $) \mid \leq 1$. In order to have more than 10 debts, on average it will take more than 10 hours. It is very rare for a connection to be silent for such a long period of time and still be considered an active connection. Thus in conclusion, normally the periodical reset of Bargain Vector is not necessary.

The bargain vector can also adapt to connection dynamics. When a new connection is accepted or an existing connection is terminated, there is no need to reset $\mathbf{B}$. The reason is that FCD always picks a connection with $\min \left\{B_{x}\right\}$, which has a significant implication for the case that a connection is terminated. For instance, let $u$ be the connection being terminated, with a bargain vector value of $B_{u}$.

- If $B_{u} \leq 0$, it implies that some of remaining active connections lost more cells for $u$, which is also reflected in $B_{x \neq u}, x \in\{1, N\}$. The selection rule of $\min \left\{B_{x}\right\}$ ensures that the remaining active connections share those $B_{u}$ losses left over by connection $u$ fairly. 
Table 1.2 Traffic types (classes) used in simulation studiesSEEE

\begin{tabular}{lllllll}
\hline Type & 1 & 2 & 3 & 4 & 5 & 6 \\
\hline Peak rate $(\mathrm{Mbs} / \mathrm{sec})$ & 10 & 5 & 2.5 & 2 & 5 & 2.5 \\
Mean rate $(\mathrm{Mbs} / \mathrm{sec})$ & 1 & 1 & 1 & 1 & 0.5 & 0.2 \\
Burst length $(\mathrm{ms})$ & 13 & 26 & 52 & 65 & 26 & 26 \\
Mean rate/Peak rate & 0.1 & 0.2 & 0.4 & 0.5 & 0.1 & 0.8 \\
$\mathrm{SCV}\left(\times 10^{2}\right)$ & 5.96 & 3.92 & 2.20 & 1.53 & 5.96 & 2.59 \\
\hline
\end{tabular}

SCV stands for Squared Coefficient of Variation of the interarrival time.

- If $B_{u} \geq 0$, it means that connection $u$ have lost more cells than its fair share. Again the selecting rule of $\min \left\{B_{x}\right\}$ allows the remaining active connections to equally benefit from it. from it.

- The value of $B_{u}$ only represents a relative measure. $B_{u} \geq 0$ does not mean that $u$ 's CLR exceeds its requirement during its life time, neither does $B_{u} \leq 0$ mean that remaining active connections' CLRs are higher than their requirements.

\section{PERFORMANCE OF FCD}

The performance of FCD have been carefully evaluated and validated via extensive simulations. In this cestion, we describe the simulation methodology and discuss the results.

\section{$3.1 \quad$ SIMULATION MODEL}

In our study, six types of On-Off sources are used and their characteristics are described in Table 1.2. The simulation runs were independently replicated 20 times, and each run included the transmission of $2 \times 10^{7}$ cells. Confidence intervals are calculated using the Student $-t$ distribution with $98 \%$ confidence. In order to perform the simulations with sufficient statistical quality and also within a reasonable time, we chose the values of desired cell loss ratio $10^{-4}, 2 \times 10^{-4}$ and $5 \times 10^{-4}$. Although $10^{-4}$ cell loss ratio is higher than that of some real applications, it does not preclude us from demonstrating the effectiveness of the method. This can be justified by Theorems I and II and was verified by experiments not shown here.

FCD's loss fairness is measured by the following error function. Let $L S_{x}$ be the number of cell losses of connection $x$ collected during the simulation life time, and $K$ be total number of simulation runs, we then 


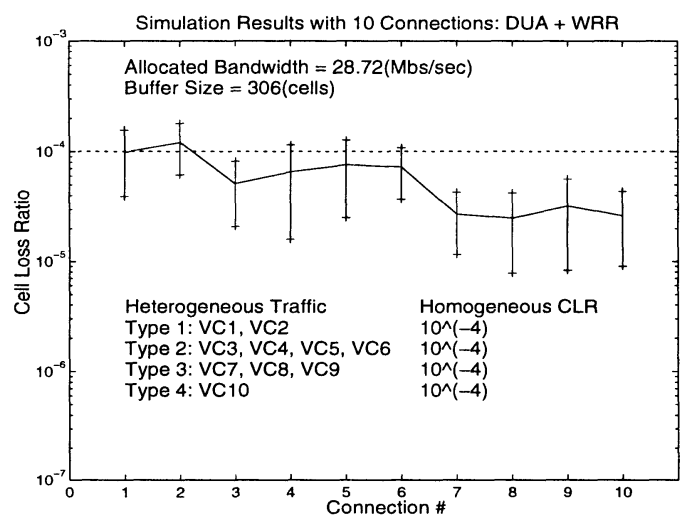

Figure 1.1 Steady state - homogeneous CLR with DUA

define an error function called Fair Error (FE) to measure the fairness performance:

$$
F E=\frac{\sum_{k=1}^{K} \sum_{x=1}^{N}\left(1-\frac{L S_{x}}{L_{x}^{f a i r} \sum_{y=1}^{N} L S_{y}}\right)^{2}}{K(N-1)}
$$

In the following sections we present the performance of FCD in two ways: (i) via monitoring its transient loss behaviours under various traffic and switch configurations; (ii) by showing long-term loss performance in terms of mean and confidence interval of CLR over the entire simulation run time. Confidence intervals are shown as vertical solid lines in the long-term figures.

\subsection{LOSS PERFORMANCE: FCD VS DUA}

We first compare the loss performance of FCD to that of DUA. The traffic configuration of simulation results reported in Figures 1.1 to 1.10 are heterogeneous. For each simulation configuration, the aggregate traffic is a random combination of various traffic types given in Table 1.2. We evaluate the FCD algorithm using both homogeneous and heterogeneous CLR requirements. The total active number of connections we used in our simulations varies from 10 to 20 . However, due to the space limitation, we only report results of 10 active connections. We should note that no matter whether the configuration is homogeneous or heterogeneous, from Property II, the resulting normalized CLR should be the same for all active connections if FCD is used.

The results of Figures 1.1 and 1.2 show that although all active connections have the same traffic load (mean rate) and the same CLR re- 


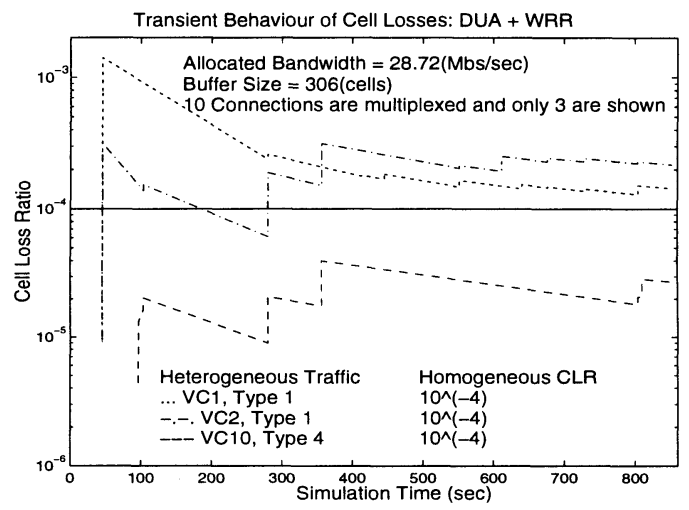

Figure 1.2 Sample path - homogeneous CLR with DUA

quirements, by using DUA, the burstier sources (i.e., VC 1 and $\mathrm{VC} 2$ ) experience a higher CLR than the less bursty connections. While the results of Figures 1.5 and 1.6 further demonstrate the fact that regardless of the CLR requirements, fot the DUA dropping policy, the actual CLR experienced by an individual connection is controlled by its burstiness and its peak rate relative to others. For instance, less bursty connections (i.e., VC 8, VC 9 and VC 10) with higher CLR requirements actually result in smaller CLRs than that of burstier ones (i.e., VC 1, VC 2, and VC 3) with lower CLR requirements. Also for the same burstiness characteristics (i.e. peak rate to mean rate ratio and squared coefficient of variation of the interarrival time of cells), if DUA is used as a dropping policy, the connections with higher peak rates (i.e. VC 1, VC 2 and VC 3) experience higher CLRs than that with lower peak rates (i.e. VC 4, VC 5, VC 6 and VC 7).

Comparing the simulation results of using FCD to these of using DUA (Figures 1.3, 1.4, 1.7, 1.8 versus Figures 1.1, 1.2, 1.5, 1.6 respectively), the effectiveness of FCD is obvious. We observe that FCD successfully breaks the "loss dependency" on traffic burstiness and regulates the loss distribution across connections according to their individual loss requirements. Here "loss dependency" is a relative term across connections rather than an absolute value of CLR. In order to understand and fully evaluate the performance of FCD both in steady state and its transient behaviour, we have constructed various configurations as shown in Table 1.4. FCD yields very satisfactory results for all cases we investigate here.

It is trivial to interpret the results where all connections have the same CLR requirements. We, thus, describe the simulation results of heterogeneous CLR requirements in greater detail. We generated 


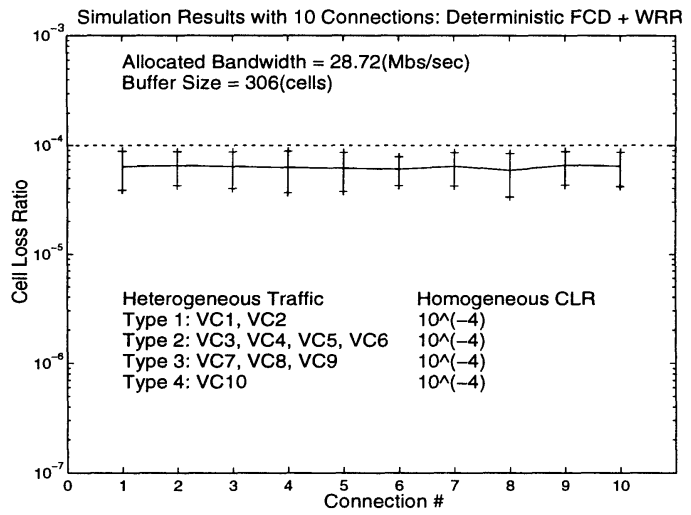

Figure 1.3 Steady state - homogeneous CLR with FCD

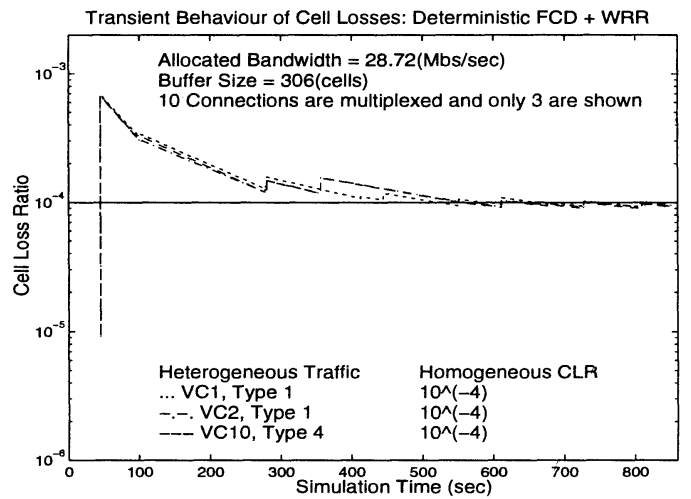

Figure 1.4 Sample path - homogeneous CLR with FCD

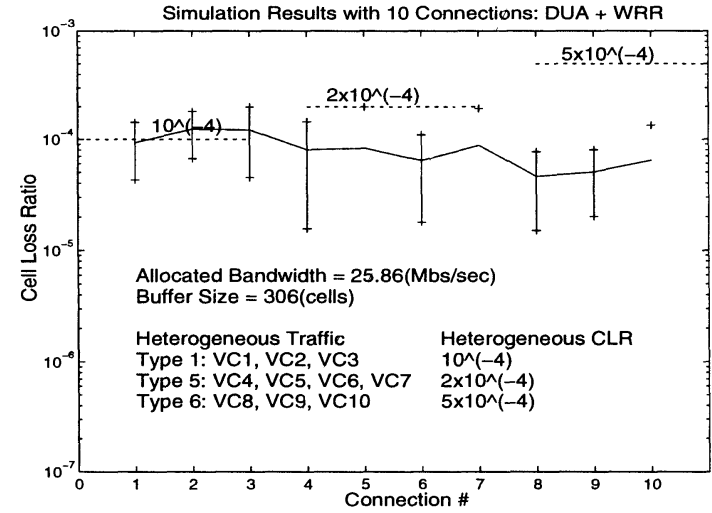

Figure 1.5 Steady state - heterogeneous CLR with DUA 


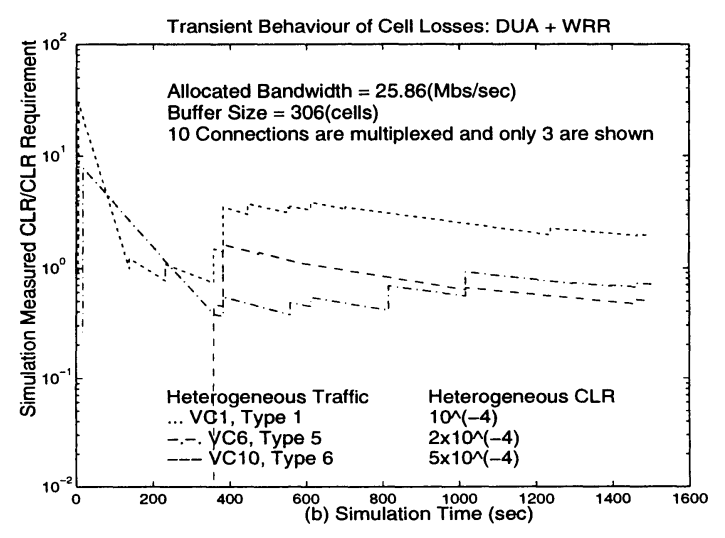

Figure 1.6 Sensitivity to heterogeneous CLR requirements - sample path heterogene ous CLR with DUA

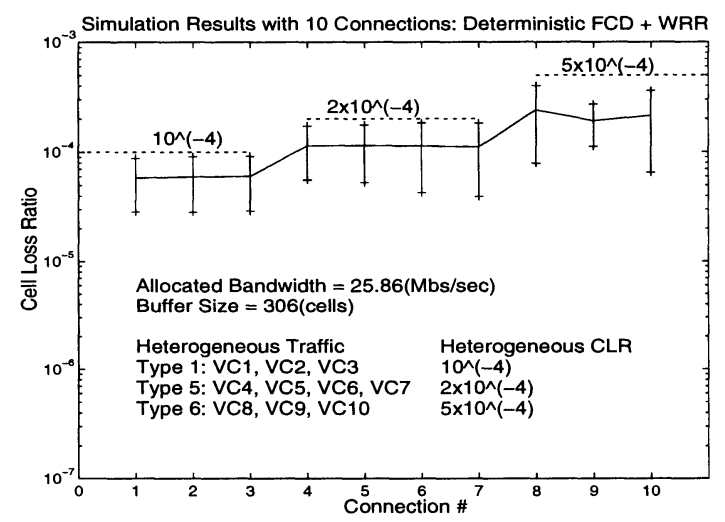

Figure 1.7 Steady state - heterogeneous CLR with FCD

Figures 1.6 and 1.8 through normalizing the sample paths of simulations for using DUA and FCD respectively. By "normalizing" we mean $\frac{\text { SimulationMeasuredCLR }}{\text { CLRReguirement }}$. From Property II, ideally all connections' normalized CLRs should be the same. Figure 1.6 simply shows the very unsatisfactory results of DUA; while Figure 1.8 demonstrates the significant effectiveness of FCD.

\subsection{SENSITIVITY TO DIFFERENT SCHEDULING ALGORITHMS}

As mentioned above, scheduling algorithms affect sub-queue length distribution whose dynamics in turn directly impact FCD's short term 


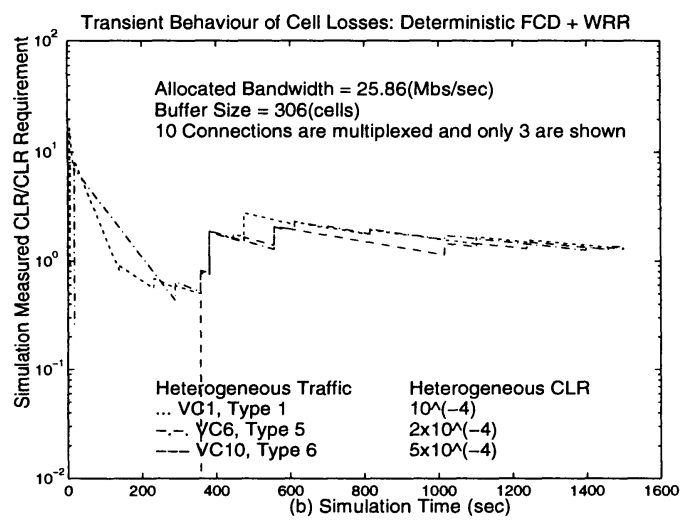

Figure 1.8 Sensitivity to heterogeneous CLR requirements - sample path - heterogen eous CLR with FCD

fairness preserved by $L_{u}^{\text {fair }}$. For instance, the probability that one finds a sub-queue length to be zero varies with different service scheduling algorithms. However, in practice, the extreme case where a chosen connection's sub-queue is always zero is very rare and should be avoided by any kind of scheduling algorithm. In our simulations we have used two kinds of service scheduling algorithms: Weighted Round Robin (WRR) and Equally Weighted Round Robin (ERR). Since our purpose here is to show differences induced by using different service scheduling algorithms, how to assign weights in WRR is not our concern in this paper. However, we do believe in assigning weights based on traffic characteristics. Also we chose to assign weights that are dramatically different from ERR so as to investigate the dependency between FCD and the service discipline. For the cases where 10 active connections are multiplexed, the weights used in simulations with homogeneous CLR requirements are given in Table 1.3 as Policy I and that used in simulations with heterogeneous CLR requirements as Policy II.

We have run simulations using both WRR and ERR. The results shown in Table 1.4 indicate that WRR always yields better performance regardless the FCD implementation - probabilistic or deterministic. Although the weights used in the service scheduling are significantly different between WRR and ERR, the resulting loss performance differences in steady state while FCD being used are negligible comparing to that of FCD vs DUA. We thus conclude that in a long run FCD is quite robust to the service scheduling. 


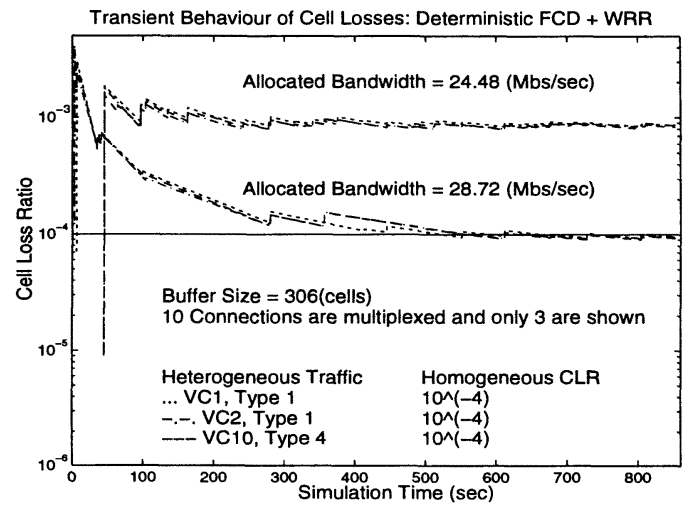

Figure 1.9 Sensitivity to bandwidth - Sample path of simulations

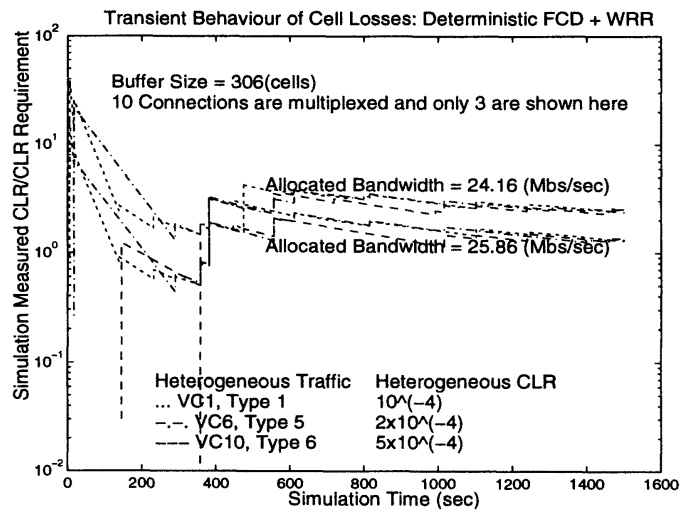

Figure 1.10 Sensitivity to heterogeneous CLR requirements and bandwidth - sample pa th of simulations 
Table 1.3 Weight Assignment for WRR Configurations

\begin{tabular}{|c|c|c|c|c|c|}
\hline & \multicolumn{2}{|c|}{ Policy I for WRR } & \multicolumn{2}{|c|}{ Policy II for WRR } & \multirow{2}{*}{$\begin{array}{l}\text { ERR } \\
\text { Weights }\end{array}$} \\
\hline & Traffic & Weights & Traffic & Weights & \\
\hline $\mathrm{VC} 1$ & Type 1 & 71 & Type 1 & 42 & 1 \\
\hline $\mathrm{VC} 2$ & Type 1 & 71 & Type 1 & 42 & 1 \\
\hline $\mathrm{VC} 3$ & Type 2 & 31 & Type 1 & 42 & 1 \\
\hline $\mathrm{VC} 4$ & Type 2 & 31 & Type 5 & 5 & 1 \\
\hline $\mathrm{VC} 5$ & Type 2 & 31 & Type 5 & 5 & 1 \\
\hline VC 6 & Type 2 & 31 & Type 5 & 5 & 1 \\
\hline $\mathrm{VC} 7$ & Type 3 & 12 & Type 5 & 5 & 1 \\
\hline $\mathrm{VC} 8$ & Type 3 & 12 & Type 6 & 1 & 1 \\
\hline $\mathrm{VC} 9$ & Type 3 & 12 & Type 6 & 1 & 1 \\
\hline VC 10 & Type 4 & 3 & Type 6 & 1 & 1 \\
\hline
\end{tabular}

Table 1.4 Various Configurations and Their Corresponding Fairness - Fair Error

\begin{tabular}{lllll}
\hline $\begin{array}{l}\text { Discarding } \\
\text { Policy }\end{array}$ & $\begin{array}{l}\text { Implementation } \\
\text { Option }\end{array}$ & $\begin{array}{l}\text { Service } \\
\text { Scheduling }\end{array}$ & $\begin{array}{l}\text { Connections } \\
\text { DUA }\end{array}$ & Connections \\
\hline FCD & Probabilistic & WRR & 0.6371601 & 0.6508583 \\
FCD & Deterministic & ERR & 0.04022239 & 0.05977245 \\
FCD & Probabilistic & WRR & 0.03158206 & 0.05036296 \\
FCD & Deterministic & WRR & 0.02873973 & 0.05061843 \\
\hline
\end{tabular}

CLR requirement is $10^{-4}$. For 10 connection cases, combination of source traffic is: 2 Type 1,3 Type 2, 4 Type 3 and 1 Type 4; link capacity $=28.72(\mathrm{Mbs} / \mathrm{sec}$ ) and buffer size $\mathrm{S}=306$ (cells). For 20 connection cases, combination of source traffic is: 8 Type 1, 6 Type 2, 3 Type 3 and 3 Type 4 ; link capacity $=52.10(\mathrm{Mbs} / \mathrm{sec})$ and buffer size $\mathrm{B}=306$ (cells).

\subsection{DETERMINISTIC IMPLEMENTATION VS PROBABILISTIC IMPLEMENTATION}

We propose two ways of implementing FCD. The simulation results of Table 1.4 suggest that deterministic implementation is a better candidate. Also we note that among all configurations which we have constructed, the best performance comes from a combination of deterministic implementation and WRR service scheduling. 


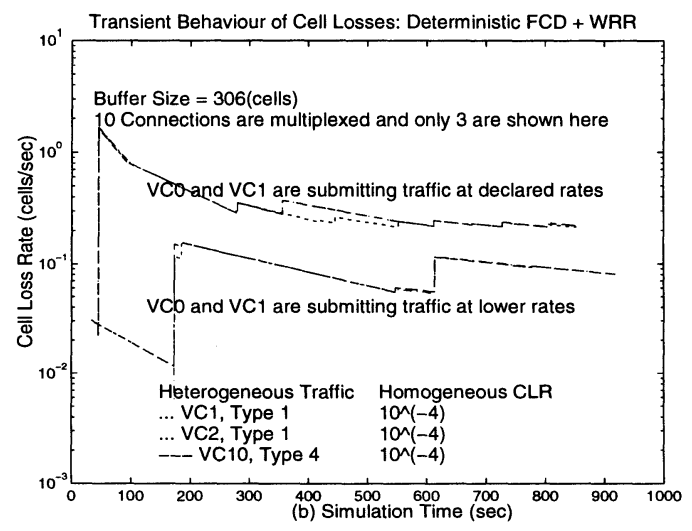

Figure 1.11 Sensitivity to load variation - Sample path of simulations

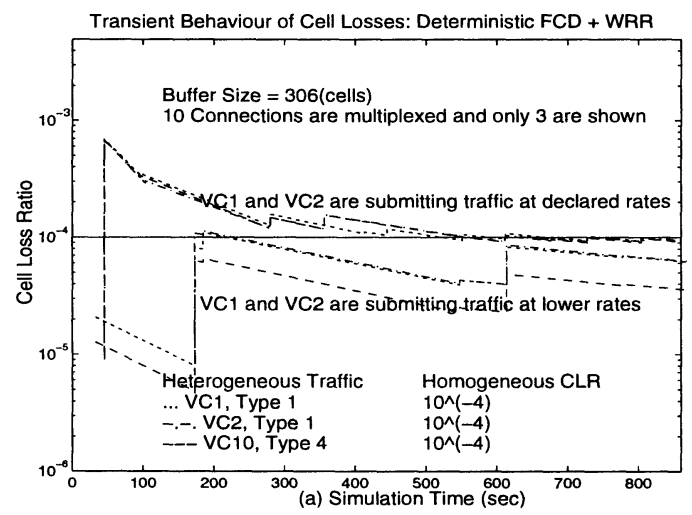

Figure 1.12 Sensitivity to load variation - Sample path of simulations

\subsection{SENSITIVITY TO LOAD VARIATION}

One of the most important aspects of any traffic control mechanisms is how to handle the traffic variation and what is the implication of its certain reactions toward traffic variation. Since we only use the mean arrival rate as the traffic input parameter in our FCD, there is no need for us to worry about the burstiness variation of the traffic. As we discussed in the previous sections, FCD is resilient to burstiness of the traffic.

Regarding the average load variation, one may face the situation where fairness related issues are heavily involved. In our study we argue that if a connection is submitting traffic at a lower rate than what it declared as long as the resulting CLRs of all connections are lower than that 


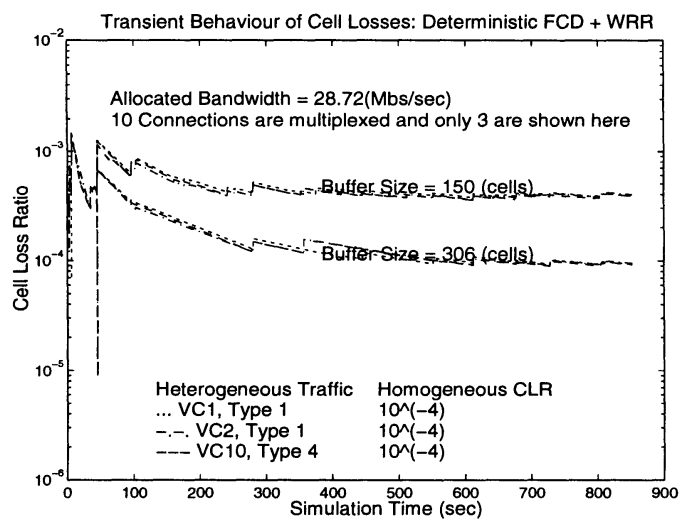

Figure 1.13 Sensitivity to buffer size - Sample path of simulations

of submitting traffic at declared rate, we state that it is fair under the condition that Property II is true.

Figures 1.12 and 1.11 present the simulation results when some connections (i.e., VC 1 and $\mathrm{VC} 2$ ) are submitting traffic at lower mean rate than their declared rates. Figure 1.12 shows us exactly what Property III predicted - (a) all connections' CLRs become lower; (b) VC 1 and VC 2 have higher relative CLRs than that of VC 10 . On the other hand Figure 1.11 indicates that all connections' actual cell loss rate in terms of "cells/sec" is decreased at the same proportion given by $\frac{P^{*}}{P}$ for $P^{*} \leq P$. Note that $P$ stands for the aggregate CLR of connections where all connections are submitting traffic at their declared rates while $P^{*}$ stands for the aggregate CLR of connections where some of the connections are submitting traffic at lower rates than what they declared.

\subsection{SENSITIVITY TO BANDWIDTH AND BUFFER ALLOCATION}

Propertys I and II state that FCD does not control the aggregate loss performance and it assures proper loss distribution across connections according to individuals' CLR requirements.

We further verify Propertys I and II via varying allocated bandwidth in our simulations. Figures 1.9 and 1.10 present several simulation sample paths of homogeneous and heterogeneous CLR requirements respectively. It is clearly shown that CLRs ofthe all active connections increase due to the decrease in allocated bandwidth and that they all increase at the same proportion. 


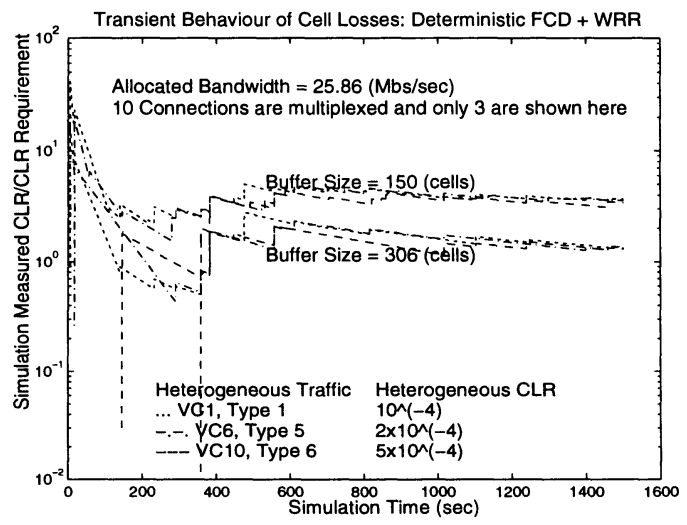

Figure 1.1/4 Sensitivity to heterogeneous CLR requirements and buffer size - sample path of simulations

FCD reacts toward decreasing/increasing the buffer size in a similar way as that of decreasing/increasing the bandwidth. Corresponding simulation results are given in Figures 1.13 and 1.14.

\subsection{SENSITIVITY TO CONNECTION LEVEL DYNAMICS}

An important issue for any traffic control scheme is how it reacts to the traffic dynamic. In above simulation studies, we evaluated FCD's sensitivity to traffic dynamic by generating bursty traffic and varying traffic load. In this section we validate FCD's robustness in response to connection-level dynamics in the following two forms: (1) impact on the remaining connections when some of the connections are terminated with non-zero values of $\mathbf{B}$ (Bargain Vector); (2) impact on existing connections when some new connections join in.

In our simulations, for the sake of simplicity, we do not re-calculate the required bandwidth when there are changes in terms of connection leaving or joining. Thus for the same buffer space and bandwidth the resulting CLRs of the remaining ones become lower after VC 1 leaves. Figure 1.15 compares CLRs of all connections in steady state for two cases: 10 connections are started and terminated together; and VC 1 is terminated earlier at 500 seconds of simulation time. Two parallel curves imply that FCD adapts very well to the connection level dynamics. This also validate our reasoning on B's robustness in Section 2.3.

Figures 1.16 and 1.17 describe the transient behaviours of some sample connections when connection VC 1 either leaves earlier or joins later during the simulation. We note that there are some jumps around 500 


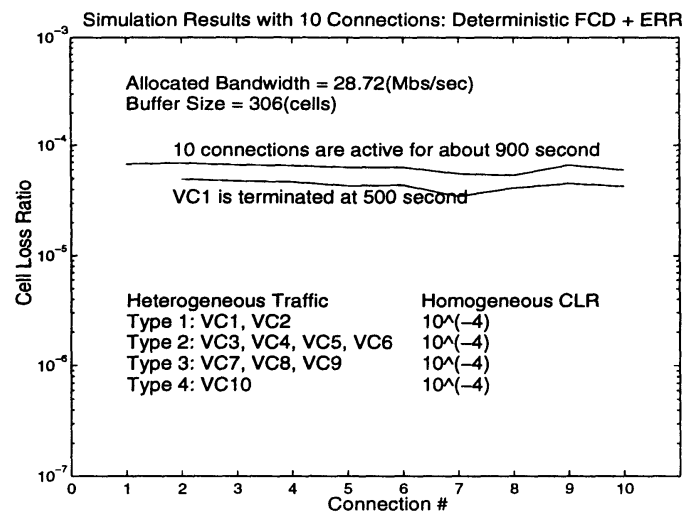

Figure 1.15 Sensitivity to connection level dynamics - steady state

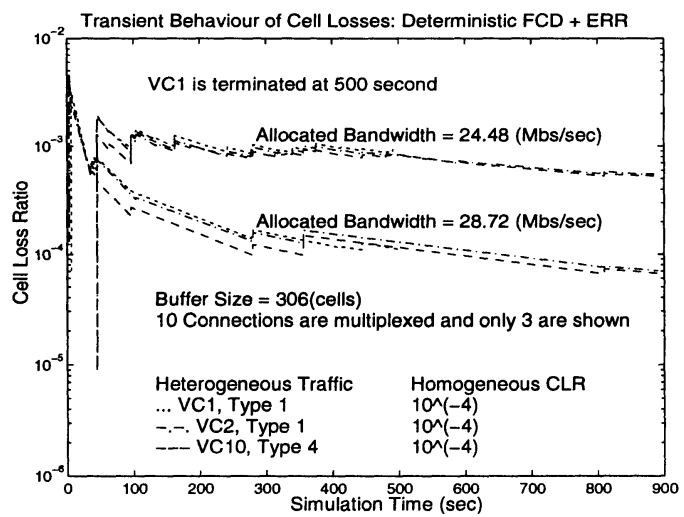

Figure 1.16 Sensitivity to connection level dynamics - Sample path of simulations 


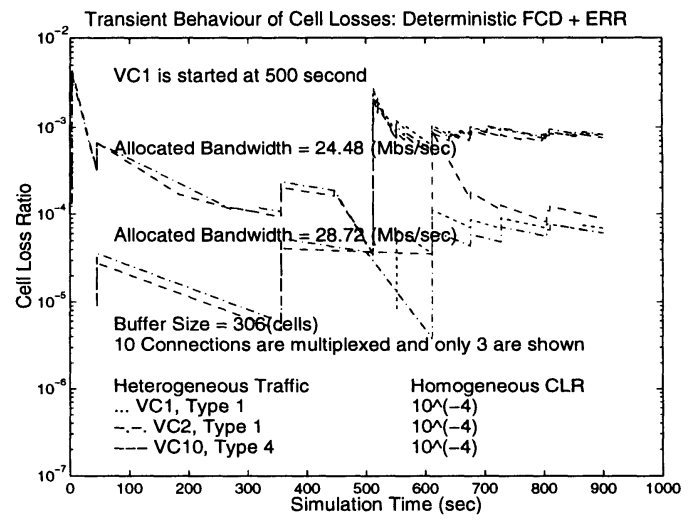

Figure 1.17 Sensitivity to connection level dynamics - Sample path of simulations with re-initialization of measurements at 500 seconds

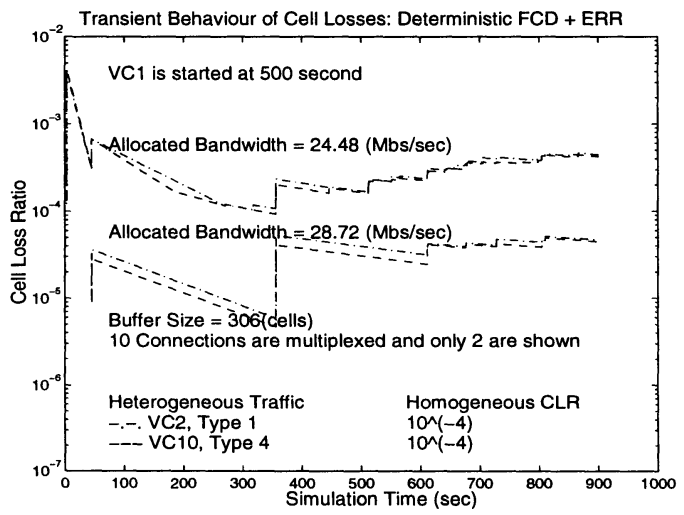

Figure 1.18 Sensitivity to connection level dynamics - Sample path of simulations without re-initialization of measurements at 500 seconds 
seconds in Figure 1.17. The reason is that we reset the simulation measurements when VC 1 starts being active around 500 seconds of simulation time. However all connections soon converge very closely after a very short period. Another way to present the same results of Figure 1.17 is to show the accumulated CLRs of VC 2 and VC 10 without reinitialization of measurements. As a result, we observe smoother curves in Figure 1.18. While for the case that VC 1 leaves earlier, there is no need to reset the measurements since all we are concerned about is whether the relative cell loss distribution across remaining active connections is kept the same. Regardless of how to present the results, the important message we obtain is that FCD can adapt very well to traffic dynamics at connection level and preserve the loss fairness given by FRL of formula (1.1).

\section{CONCLUSIONS}

Finally we conclude our study and highlight our findings:

- Simulations results confirm the theoretical findings that FCD provides a robust mechanism to allocate cell loss to diverse connections in a fair manner.

- FCD does not require prior knowledge of detailed traffic characteristics and only mean rate is needed, which can be declared by connections at setup time or estimated during the connection life time.

- FCD is a solution for heterogeneous environment in terms of both traffic characteristics and QoS requirements.

- In the long run (i.e. accumulated number of losses exceed 100 cells), FCD is insensitive to which service scheduling algorithm is being used and how it is implemented in terms of static vs probabilistic.

- Scheduling has a direct impact on short term performance.

- The FCD algorithm is very simple and can be implemented without incurring too much overhead. It is only active when total buffer occupancy reaches its limit.

- Deterministic implementation of FCD outperform a probabilistic one in terms of resulting values of $\mathrm{FE}$, i.e. $F E($ det. $)<F E($ prob. $)$.

- FCD is a robust scheme in the sense that it can co-exist with various resource allocation and scheduling schemes, which provides 
us higher flexibility to deal with complicated ATM traffic management issues and allows us to decouple the methods for addressing those issues.

\section{Appendix: Mathematical Proofs}

\section{Proof of Property II}

Since for given buffer size and traffic characteristics, if allocated bandwidths are $C_{1}>C_{2}$, the resulting aggregate CLR $P\left(C_{1}\right)<P\left(C_{2}\right)$, thus for $u \in\{1, N\}$

$$
\frac{p_{u}\left(C_{1}\right)}{p_{u}\left(C_{2}\right)} \approx \frac{P\left(C_{1}\right) L_{u}^{\text {fair }}}{P\left(C_{2}\right) L_{u}^{\text {fair }}}=\frac{P\left(C_{1}\right)}{P\left(C_{2}\right)}<1
$$

with equality when $B_{u}=0$.

\section{Proof of Property III}

If a subset of connections $\mathbf{V} \subseteq \mathbf{U}$ submits traffic at a lower mean rate, $\lambda_{v}^{*}$, than their declared rate, $\lambda_{v}$, i.e. $\lambda_{v}^{*}<\lambda_{v}$, the resulting total mean arrival rate is $\lambda^{*}<\lambda$ and the corresponding aggregate cell loss ratio is $P^{*}<P$. We thus have

$$
\begin{gathered}
\lambda^{*}=\lambda-\sum_{v} \lambda_{v}+\sum_{v} \lambda_{v}^{*} \\
p_{v}^{*}=\frac{\lambda^{*} P^{*} \lambda_{v} p_{v}}{\lambda_{v}^{*} \lambda P}
\end{gathered}
$$

- Let $V$ be a particular connections of subset $\mathbf{V}$, for connection $V$, what we want to prove is $p_{V}^{*}<p_{V}$ or

$$
\frac{\lambda^{*} \lambda_{V}}{\lambda_{V}^{*} \lambda} \frac{P^{*}}{P}<1
$$

Since $P^{*}<P$, as long as the following inequality (1.A.5) is true

$$
\frac{\lambda^{*} \lambda_{V}}{\lambda_{V}^{*} \lambda}<1 \quad \text { or } \quad \lambda_{V}^{*} \lambda-\lambda_{V} \lambda^{*}<1
$$

the inequality of (1.A.4) will be true. From (1.A.2) we have

$$
\lambda^{*}=\lambda-\sum_{v \neq V} \lambda_{v}+\sum_{v \neq V} \lambda_{v}^{*}-\lambda_{V}+\lambda_{V}^{*}
$$


and it is trivial to prove the following inequality

$$
\begin{gathered}
\lambda_{V}^{*} \lambda-\lambda_{V} \lambda^{*}<\lambda_{V}^{*} \lambda-\lambda_{V}\left(\lambda^{*}+\sum_{v \neq V}\left(\lambda_{v}-\lambda_{v}^{*}\right)\right) \\
=-\left(\lambda_{V}-\lambda_{V}^{*}\right)\left(\lambda-\lambda_{V}\right)=-\left(\lambda-\lambda_{V}\right)^{2}<1
\end{gathered}
$$

- For $\forall w \notin \mathbf{V}$, we have

$$
p_{w}^{*}=\frac{\lambda^{*} P^{*} \lambda_{w} p_{w}}{\lambda_{w} \lambda P}=\frac{\lambda^{*} P^{*} p_{w}}{\lambda P}<\frac{\lambda P^{*} \lambda_{w} p_{w}}{\lambda_{w} \lambda P}=\frac{P^{*} p_{w}}{P}<p_{w}
$$

- From (1.A.3) and (1.A.6), we have

$$
\frac{p_{w}^{*}}{p_{w}}-\frac{p_{v}^{*}}{p_{v}}=\frac{\lambda^{*} P^{*}}{\lambda P}-\frac{\lambda^{*} P^{*} \lambda_{v}}{\lambda P \lambda_{v}^{*}}=\frac{\lambda^{*} P^{*}}{\lambda P}\left(1-\frac{\lambda_{v}}{\lambda_{v}^{*}}\right)<0
$$

Thus

$$
\frac{p_{w}^{*}}{p_{w}}<\frac{p_{v}^{*}}{p_{v}}
$$

Property IV can be proved similarly.

\section{Acknowledgment}

We are grateful for discussions with Arthur Berger and his review of an early draft of this paper.

Xiaowen Mang received BSEE from Beijing University of Posts and Telecommunications (Beijing, China), DEA (Diplôme d'Etudes Approfondies) from University of Paris VI (Paris, France) and Ph.D. from Duke University (North Carolina, USA). She is now a senior technical staff member in the Networl Design and Performance Analysis Department at AT\&T Labs.

Yonatan (Yoni) Levy is a technical manager in the Network Design and Performance Analysis Department at AT\&T Labs. He received M.S. in Operations Research in 1973 from Tel-Aviv University and Ph.D. in Mathematical Sciences from the Johns Hopkins University in 1980. His interests include the design, analysis, and optimization of telecommunication networks and services, with current focus on efficient delivery of QoS. Yoni holds two patents on network call distribution.

\section{References}

Y. Arian and Y. Levy (1992). Algorithms for generalized round robin. Operations Research Letters, 12:313-9.

S. Archambault and J. Yan (1996). Performance analysis of per-vc queueing. Proc. GLOBECOM'96, pages 1721-25. 
A. Choudhury and E. Hahne (1996). Dynamic queue length thresholds in a shared memory. Proc. INFOCOM'96, pages 679-87.

B. Collier and H. Kim (1996). Efficient analysis of shared buffer management strategies in atm network under non-uniform bursty traffic. Proc. INFOCOM'96, pages 671-8.

A. Conway and C. Rosenberg (1996). Weighted fair blocking mechanism for discrete-time multiplexing. Proc. INFOCOM'96, pages 1045-53.

G. Chen and I. Stavrakakis (1996). Atm traffic management with diversified loss and delay. Proc. INFOCOM'96, pages 1037-44.

A. Demers, S. Keshav, and S. Shenker (1989). Analysis ans simulation of a fair queueing algorithm. SIGCOMM'89, pages 1-12.

S. Golestani (1994). A self-clocked fair queueing sceheme for broadband applications. Proc. INFOCOM'94, pages 636-46.

D. Heyman, A. Tabatabai, and T. Lakshman (1992). Statistical analysis and simulation study of video teleconference traffic in atm networks. IEEE Trans. Circuits and Sys. for Video Technology, 2(1):49-59.

K. Kawahara, K. Kitajima, T. Takine, and Y. Oie (1996). Performance evaluation of selective cell discard schemes. Proc. INFOCOM'96, pages 1055-61.

S. Lee, D. Cho, and Y. Park (1996). Improved dynamic weighted cell scheduling algorithm based on earliest deadline first scheme for various traffics of atm switch. Proc. GLOBECOM'96, pages 1959-63.

X. Mang and E. Gelenbe (1996). Call admission control in ATM using the diffusion model. Proc. GLOBECOM'96, pages 1700-1704, 1996.

A. Parekh and R. Gallager (1993). A generalized peocessor sharing approach to flow control in integrated services networks - the multiple node case. Proc. INFOCOM'93, pages 521-30.

Y. Takagi, S. Hino, and T. Takahashi (1991). Priority assignment control of ATM line buffers with multiple qos classes. IEEE J. SAC, SAC9(7):1078-1092, September 1991.

D. Wilson and M. Ghanbari (1996). An efficient loss priority scheme for mpeg-2 variable bit rate video for atm networks. Proc. GLOBECOM'96, pages 1954-8.

T. Yang and J. Pan (1996). A measurement-based loss scheduling scheme. Proc. INFOCOM'96, pages 1062-71. 


\section{Tools and Techniques}

